in the inflammatory biomarkers (IL-4, IL-6, hs-CRP, TNF- $\alpha$, TGF- $\beta$ and mean ESR between the curcuminoid treatment group and the placebo group $(p>0.05)^{6}$.

Conclusion: Enriched boswellic acid and curcumin/piperine formulations demonstrate efficacy and safety for suitable treatment option: both ingredients, often cited as natural alternatives to address OA pain and stiffness could be evaluated to explore the potential benefit as a formulated combination.

REFERENCES:

[1] Vishal et al. Int. J. Med. Sci. 2011,8

[2] Sengupta et al. Int. J. Med. Sci. 2010, 7

[3] Sengupta et al. Mol Cell Biochem. 2011, 354:189-197.

[4] Shoba et al. Planta Med. 1998 May;64(4):353-6

[5] Panahi et al. Phytother. Res. 28: 1625-1631 (2014).

[6] Rahimnia A-R et al. Drug Res 2015; 65: 521-525.

Disclosure of Interests: Vidhu Sethi Employee of: Employee of GSK Consumer Healthcare, Kamran Siddiqui Employee of: Employee of GSK Consumer Healthcare, Manohar Garg: None declared.

DOI: 10.1136/annrheumdis-2021-eular.2247

\section{AB0596 \\ THE POSSIBLE CONTRIBUTION OF DEHYDROEPIANDROSTERONE SULFATE FOR OSTEOARTHRITIS OF THE KNEE}

I. Bashkova ${ }^{1,2}$, I. Madyanov ${ }^{1,3}$, K. Misko ${ }^{1}$, E. Preobrazhenskaia ${ }^{4} .{ }^{1}$ Chuvash State University named after I.N. Ulyanov, Department of Hospital Therapy,

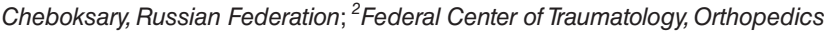
and Endoprosthesis, Consultative polyclinic, Cheboksary, Russian Federation; ${ }^{3}$ Republican Clinical Hospital, Department of Endocrinology, Cheboksary, Russian Federation; ${ }^{4} \mathrm{Federal}$ Center of Traumatology, Orthopedics and endoprosthesis, Scientific and Educational Department, Cheboksary, Russian Federation

Background: Genetic, biochemical, metabolic, hormonal (primarily imbalance of sex hormones) factors are involved in the progression of osteoarthritis (OA). Dehydroepiandrosterone and its metabolite, dehydroepiandrosterone sulfate (DHEA-S), have a stress-limiting, anti-atherogenic, anti-diabetic, antihypertensive, anti-infective, immunomodulatory effects. Heroprotective effect is not excluded. Experimental studies have identified a relationship between an age-related decrease in DHEA-S levels and various adverse effects of aging.

Objectives: To identify the contribution of DHEA-S to the pathogenesis of OA, it's advisable to conduct a comparative analysis of connection of the adrenal hormones with clinical, laboratory, radiological signs of the course of OA.

Methods: Patients with primary OA with a lesion of the knee joints ( $n=90$, including 22 men) were examined. The age of the patients - 29-69 years, the duration of the disease $-1.5-20$ years. The control group ( $n=114$, including 26 men) was formed by random sampling of the population from healthy people, it's representative by gender and age. We investigated the serum levels of cortisol, DHEA-S, estradiol (in women), testosterone (in men) and carried out radiography of the knee joints. OA was diagnosed using R.D. Althman, the x-ray stage-according to the classification of Kellgren and Lawrence. Statistically determined the mean value, standard deviation. Differences between the samples were considered statistically significant at $p<0.05$. To create a model of OA pathogenesis, the method of principal components of factor analysis was used.

Results: The DHEA-S level in the blood of patients with OA was lower than that of the control group $(2.40 \pm 1.20$ vs $3.66 \pm 1.45 \mu \mathrm{g} / \mathrm{ml}, \mathrm{p}=0.001)$, in women-was lower, than in men $(2.25 \pm 1.17$ vs $2.89 \pm 1.23 \mu \mathrm{g} / \mathrm{ml}, \mathrm{p}=0.045)$. In the control group, gender differences were not statistically significant $(p>0.05)$. All patients with $O A$ showed an inverse correlation between age and DHEA-S $(r=-0.511, p=0.0001$, and $r=-0.549, p=0.0001$ respectively). For factor analysis the most important signs for the course of OA are ESR levels, C-reactive protein (CRP) in the blood (as markers of inflammatory component of $\mathrm{OA}$, or factor 2) were selected, and the radiological stage of OA (degenerative component, or factor 1). In women, we regarded factor 1 as "degenerative", the maximum contribution to total dispersion was made by the «x-ray stage» $(+0.72)$. This symptom was opposed by the «DHEA-S level» $(-0.79)$ and «estradiol blood level» (-0.68), which suggests a link between degenerative and dystrophic processes in the knee joint in women with OA and a decrease in blood levels of DHEA-S and estradiol. Factor 2 we interpreted as «inflammatory». This was indicated by the values of "CRP» $(+0.66)$ and «ESR» $(+0.64)$. The «inflammatory» factor in women from hormonal indicators was opposed by the «blood cortisol content» $(-0.31)$ and «DHEA-S level» $(-0.26)$. Factor 1 in men accounted for $46 \%$ of the total variance. Since factor 1 in men included the most significant «CRP» $(+0.85)$, "X-ray stage» $(+0.77)$ and «ESR» (+0.72), we called it «antidegenerative anti-inflammatory factor». The maximum value (modulo) in factor 1 is for DHEA-S $(-0.76)$ and the lower is for testosterone $(-0.53)$. So, in men, a sufficient level of DHEA-S is closely related to the "antidegenerative-anti-inflammatory" factor of OA pathogenesis and DHEA-S counteracts 2 key pathogenetic processes simultaneously-degenerative and inflammatory.

Conclusion: In women, a decrease in DHEA-S is a risk factor for the predominantly degenerative component of $\mathrm{OA}$, in men it's a universal risk factor, predisposing both to the development of inflammation and degenerative changes in the joints

Disclosure of Interests: None declared.

DOI: 10.1136/annrheumdis-2021-eular.2308

\section{AB0597 COMPLIANCE WITH CLINICAL PRACTICE GUIDELINES IN KNEE OSTEOARTHRITIS}

M. Yasmine ${ }^{1}$, L. Souebni ${ }^{1}$, S. Miladi ${ }^{1}$, A. Fazaa ${ }^{1}$, S. Mariem ${ }^{1}$, K. Ouenniche ${ }^{1}$, S. Kassab ${ }^{1}$, S. Chekili ${ }^{1}$, K. Ben Abdelghani ${ }^{1}$, A. Laatar ${ }^{1}{ }^{1}$ Mongi Slim Hospital, Rheumatology, Tunis, Tunisia

Background: Knee osteoarthritis $(\mathrm{OA})$ is a leading cause of disability among olde adults. Recently, evidence-based guidelines for the comprehensive management of osteoarthritis (OA) were developed by the American College of Rheumatology (ACR). Objectives: The aim of this study was to assess compliance of doctors with ACR 2019 clinical practice guidelines for the management of knee OA.

Methods: We conducted a prospective study including rheumatologists and general practitioners. The doctors were invited to answer a structured questionnaire via Google Form. The outcomes of interest concerned the medical management of knee $\mathrm{OA}$ as well as alternative medicine.

Results: The study included 100 doctors: 75 rheumatologists and 25 general practitioners. Almost half of them (49\%) have been practicing medicine for more than 10 years. Forty four percent of doctors see between 10 to 20 patients with knee OA per month and $47 \%$ of them declared seeing more than 20 patients. Regarding the pharmacological treatment of knee OA flares, oral Non-steroidal Anti-inflammatory drug (NSAIDs) was the initial molecule of choice $(91 \%)$ followed by grade I analgesics $(86 \%)$ and topical NSAIDs $(68 \%)$. Tramadol and non-Tramadol opioids as well as intraarticular glucocorticoid injections were prescribed respectively in $41 \%$ and $46 \%$ of cases. Glucosamine and chondroitin sulfate were prescribed in $49 \%$ and $54 \%$ of cases respectively and as a combination in $20 \%$ of cases. The reasons for non-prescribing these molecules were non-affordable prices $(n=19)$, a lack of efficacy $(n=6)$ and potential sides effects $(n=1)$. Seventy three percent of doctors prescribe hyaluronic acid injections, with a frequency of three weekly injections in $38.7 \%$ of cases and according to the response to the first injection in $61.3 \%$ of cases. The combination of both corticosteroids and hyaluronic acid injection was preferred in $38 \%$ of cases. The majority of doctors $(84 \%)$ referred their patients to physical therapy as a first-line prescription $(82.1 \%)$ or after medical treatment failure $(17.9 \%)$. The use of alternative medicine was at follows: acupuncture $(42.7 \%)$ prolotherapy $(28.1 \%)$ and platelet-rich plasma injections (16.7\%). Thirty eight doctors recommended against alternative medicine.

Conclusion: Our study showed a poor compliance to guidelines regarding the use of intra-articular injections and alternative medicine. Even though, these guidelines provide direction for clinicians, doctors and patients should engage in shared decision-making that accounts for patients' values, preferences, and susceptibilities. Disclosure of Interests: None declared.

DOI: 10.1136/annrheumdis-2021-eular.2896

\section{AB0598 EFFICACY OF ULTRASOUND GUIDED INJECTIONS OF A CROSS-LINKED SODIUM HYALURONATE COMBINED WITH TRIAMCINOLONE HEXACETONIDE FOR OSTEOARTHRITIS OF THE KNEE}

F. Porta ${ }^{1}$, G. Filippou ${ }^{2}$, G. Sakellariou ${ }^{3} .{ }^{1}$ Florence, Istituto di Neuroscienze, Florence, Italy; ${ }^{2}$ Luigi Sacco University Hospital, Rheumatology Department, Milano, Italy; ${ }^{3}$ University of Pavia, Istituti Clinici Scientifici Maugeri IRCCS, Pavia, Italy

Background: According to guidelines, the use of steroid and/or hyaluronate (HA) intra-articular injections for knee osteoarthritis (KOA) is controversial. Heterogeneity of studies and difference in HAs characteristics does not allow to draw safe conclusions. One of the major concerns is the accuracy of the procedure as up to $1 / 3$ of injections could miss joint space when performed blindly (1), negatively affecting the efficacy of HA that needs to be placed correctly in the joint space Objectives: The aim of our study was to evaluate the longterm efficacy of a novel association of a Cross-Linked Sodium Hyaluronate Combined With Triamcinolone Hexacetonide (SHCTH) in patients with KOA in a real life setting. Methods: We retrospectively evaluated the clinical and ultrasonographic (US) data of patients (pts) affected by symptomatic KOA with intra-articular injections of SHCTH (1 injection every 6 months). Pts with concomitant inflammatory arthropaties were excluded. US guidance was carried out with the "in plane" technique choosing either the lateral suprapatellar or midpatellar approach. All pts were evaluated for pain with a VAS 0-10 for pain at baseline and after 2 weeks, 1 3, 6, 9 and 12 months, with the WOMAC questionnaire and with US, scoring joint effusion, synovial hypertrophy (SH) and power Doppler (PD) synovial signal. Due to the retrospective design, the WOMAC data were available as VAS or Likert scales; to allow comparability these values were standardized. Clinical and US variables at different time points were compared using the Wilcoxon rank sing test, the McNemar test or the paired samples t-test, depending on the variable. 